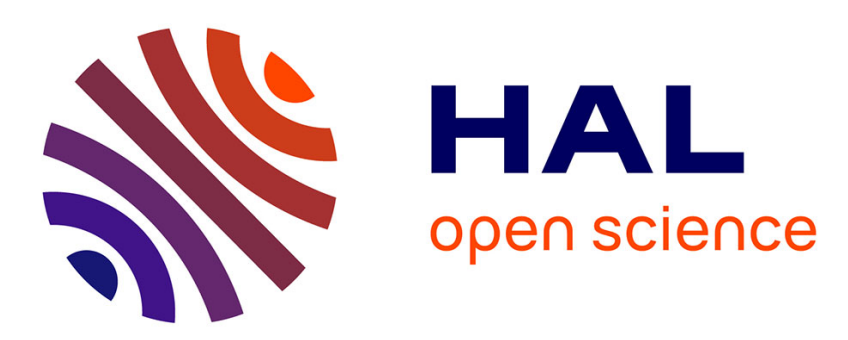

\title{
A novel design method for the fast and cost-effective manufacture of composite parts employing the Quilted Stratum Process
}

François-Xavier Irisarri, Terence Macquart, Cédric Julien, Denis Espinassou

\section{- To cite this version:}

François-Xavier Irisarri, Terence Macquart, Cédric Julien, Denis Espinassou. A novel design method for the fast and cost-effective manufacture of composite parts employing the Quilted Stratum Process. Composites Part B: Engineering, 2018, 158, pp.364-372. 10.1016/j.compositesb.2018.09.070 . hal02317960

\author{
HAL Id: hal-02317960 \\ https://hal.science/hal-02317960
}

Submitted on 16 Oct 2019

HAL is a multi-disciplinary open access archive for the deposit and dissemination of scientific research documents, whether they are published or not. The documents may come from teaching and research institutions in France or abroad, or from public or private research centers.
L'archive ouverte pluridisciplinaire HAL, est destinée au dépôt et à la diffusion de documents scientifiques de niveau recherche, publiés ou non, émanant des établissements d'enseignement et de recherche français ou étrangers, des laboratoires publics ou privés. 


\title{
A novel design method for the fast and cost-effective manufacture of composite parts employing the Quilted Stratum Process
}

\author{
F.X. Irisarri ${ }^{\mathrm{a}}$, T. Macquart ${ }^{\mathrm{b}}$, C. Julien ${ }^{\mathrm{a}}$, D. Espinassou ${ }^{\mathrm{c}}$ \\ ${ }^{a}$ DMAS, ONERA, Université Paris Saclay, F-92322 Châtillon, France, francois-xavier.irisarri@onera.fr, cedric.julien@onera.fr \\ ${ }^{b}$ Bristol Composite Institute (ACCIS),University of Bristol, England, terence.macquart@bristol.ac.uk \\ ${ }^{c}$ CETIM - Technical Centre for Mechanical Industry, Chemin du Chaffault 44340 Bouguenais, France, denis.espinassou@cetim.fr
}

\begin{abstract}
Composite materials, and carbon fiber reinforced plastics (CFRP) in particular, are nowadays widely used in the design of high performance aeronautical and aerospace lightweight structures. Applications to other sectors have, however, been limited due to their high costs and long processing times. To address these issues, the CETIM has developed the Quilted Stratum Process (QSP); a fully automated high-volume production line of thermoplastic composite parts. The QSP manufactures variable-thickness and variable-stiffness composite laminates by assembling composite layers of various orientations and shapes. The focus of this work is to bring together the great versatility of composite design offered by the QSP under a single coherent framework, automating and simplifying the design of QSP-manufactured structures. To this end, we develop the Quilted Stratum Design (QSD). The QSD is a three-step laminate optimization procedure during which the structure is first ideally optimized, then simplified and finally detailed layer by layer to be interpreted for QSP-manufacturing. The method is presented in this paper and applied to both an academic and an industrial case study for which the trade-off between the manufacturing complexity and mechanical performances is studied. In both our applications, partitioning the structure into small numbers of constant stiffness areas was found to be sufficient to significantly over-perform conventional constant stiffness designs and well-compatible with the QSP.
\end{abstract}

Keywords: A. Layered structures, A. Thermoplastic resin, A. Discontinuous reinforcement, B. Buckling, Variable-stiffness optimization

\section{Introduction}

Composite materials, and carbon fiber reinforced plastics (CFRP) in particular, are nowadays widely used in the design of high performance aeronautical and aerospatial lightweight structures. Besides these flagship applications, other industries such as offshore oil, wind energy, and automotive have also found numerous applications availing to the high specific stiffness and strength-to-weight ratios of composite materials. Compared to light metal alloy, CFRPs also offer additional structural degrees of freedom, related to the fiber orientations. The resulting increase in design freedom has been and still is responsible for much of the enthusiasm given to composite laminate optimization in the literature; a review of which can be found in [1, 2]. 
The mechanical properties of composite laminates are characterized by their constituents, e.g. fiber and matrix materials, but also by their manufacturing process which influences fiber-matrix adhesion, interlaminar strength and residual stresses. From a design point of view, the manufacturing process defines and constrains the design space, generally trading-off complexity for reduced manufacturing costs. Consequently, many design and optimization methods for composite structures are specific to manufacturing processes.

In the aeronautic industry, the design of classical laminates manufactured by hand lay-up or automated tape placement processes is subjected to numerous design guidelines motivated either by the manufacturing process itself or by past experiences about the mechanical behavior of composite laminates [3, 4]. Designing a single laminate is known in the literature as a constant-stiffness design problem. Laminate design guidelines such as symmetry and balance of the laminate have been considered since the very first developments about constant-stiffness optimization [5-7].

Contrary to constant-stiffness structures, variable-stiffness structures consist of multiple stacking sequences. Large composite panels with thickness variations obtained by adding or terminating plies form a first class of variable-stiffness structures. The design of such panels is commonly referred to as the laminate blending optimization problem [8-11]. Specific developments about handling ply-drop design guidelines can be found in [12, 13].

The development of Automated Fiber Placement (AFP) machines has driven a significant research effort to devise specialized optimization methods. Contrary to conventional laminates, AFP processes enable continuous variation of the fiber orientation within each layer. This results in an enlarged design space for variable-stiffness design whose exploration requires specialized optimization methods. Numerous direct parameterizations of the tow paths have been proposed, for instance in [14-20].

The most advanced methods for variable-stiffness design combine, within multi-step approaches, gradient based optimization and evolutionary algorithms for their complementary efficiency in solving continuous and combinatorial problems respectively. Lamination parameters are used to find the optimal stiffness distributions in combination with Finite Element (FE) analyses [21-25]. Alternatively, the polar parameterization can also been used by [26-28] for this purpose. The subsequent optimization steps aim at retrieving the corresponding fiber angle distributions [29] and tow path [30] while taking into account manufacturing constraints [31].

The present work is a first step towards devising a composite structure design method specialized for the Quilted Stratum Process (QSP) developed by the CETIM [32]. The QSP, in short, is a multi-step process transforming raw materials (e.g. fibres and resin) into sophisticated composite parts, providing an economically viable solution for high volume production. QSP structures are built-up from an assembly of composite patches. A patch is a composite layer whose material, size, shape, orientation, position and stacking order in the assembly are design variables. Assembling patches enables piecewise linear fiber orientation variations as well as thickness variations. As such, QSP structures constitute a specific class of variable-stiffness structures.

The optimization of laminated structures made of patches has been studied by Zehnder and Ermanni in [33] 
and further developed by Giger et al. [34]. Both studies focus on devising an appropriate parametrization to solve the optimization problem using a genetic algorithm. Such an approach, however, entails computational costs incompatible with time and cost constraints of most design offices. In this study, in order to better control the computational costs, a multi-step design procedure is proposed, derived from variablestiffness methods.

From the general presentation of the QSP in Section 2, degrees of freedom and constraints imposed by the manufacturing process on the design of composite structures are determined. Section 3 presents the proposed three-step Quilted Stratum Design (QSD) method. The first step starts with a variable thickness and stiffness structural optimization employing lamination parameters. In the subsequent step, the design is simplified into a reduced number of constant-stiffness zones. The last step aims at retrieving the fiber orientations per zone in order to sketch out a design that can be manufactured using the QSP. Results of two design case studies are presented and discussed in Section 4 whilst concluding remarks are given in Section 5

\section{The Quilted Stratum Process design problem}

The use of CFRP composites in mass-production vehicles remains scarce, mostly due to their high costs and long processing times. Reducing material and manufacturing costs as well as increasing production rates are key issues that must be addressed before the automotive industry can fully integrate CFRPs within mass-produced vehicles. Aeronautic manufacturers face similar challenges for parts such as seat structures, window frames, or FAN blades. The CETIM, in an effort to address these challenges, has developed the Quilted Stratum Process (QSP).

The QSP, presented in Figure 1, is a fully automated four-step process transforming raw materials (i.e. fibers and resin) into complex composite parts in a short turnaround time. The production line starts with the continuous pultrusion of thermoplastic composite tapes that feeds into a cutting module. Alternately, any thermoplastic composite tape avaible from material suppliers can be used. Ultrasonic blades are used to cut the tape into patches of variable shapes. Patches are then picked from carriers and placed and welded in precise position on a conveyor system. Patches can be joined end to end to form composite layers with piecewise linear fiber orientation variations. The layers are laid in successive assembly stations to build up a variable-thickness preform on a flat surface tool. The preform is then heated using a combination of infrared and conduction heating devices in order to provide sufficient energy to the thickest areas without degrading the thinnest areas. Finally the preform is thermo-formed in a hot press and demolded. The QSP minimizes material waste and produces net-shape parts with short processing time, thus providing an economically viable solution for high volume production. The cycle time is driven by the press cycle which varies form one to ten minutes depending on the maximal thickness of the part (up to $6 \mathrm{~mm}$ thick) and the materials. Net shape parts can be obtained by simultaneous thermoforming and overmolding of the part edges. A large variety of inserts can also be added in the same press-forming cycle. 


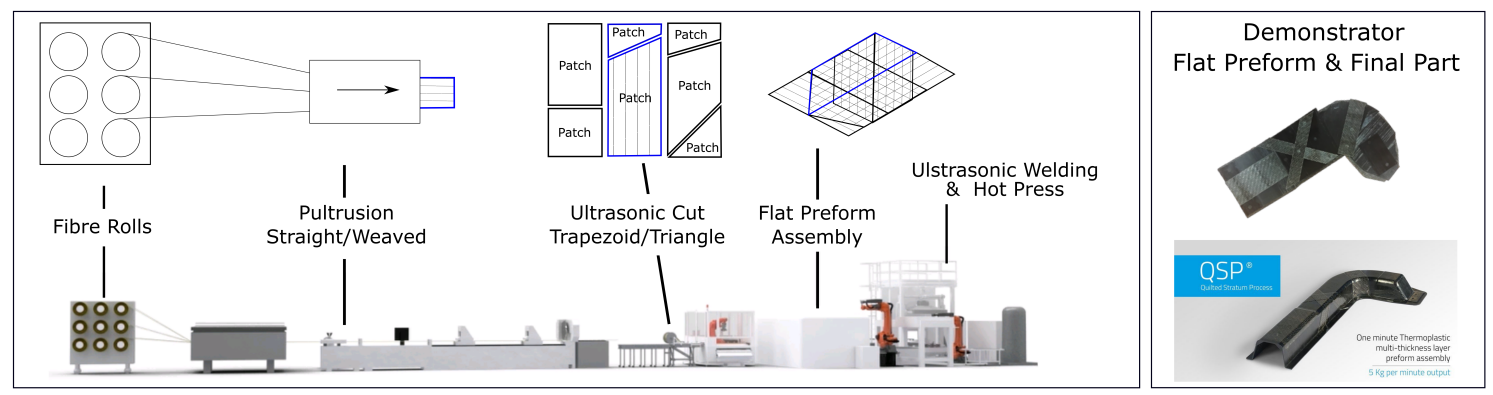

Figure 1: The Quilted Stratum Process

From a design viewpoint, the key issue is the patch assembly. The assembly is defined by multiples patches, positioned, rotated, and stacked on a flat surface, to obtain a preform. Additionally, the QSP is a versatile process which enables numerous variations in manufacturing patches which must also be considered, thus a patch is defined by a shape, a size, a position, a material and its orientation. The degrees of freedom listed above form an extremely large and complex design space due to their number and diverse nature. Direct sampling and search in such a design space could be very costly in terms of computational cost. This problem is thoroughly discussed in [33, 34]. Thus, in the following, a multi-step approach is proposed. As this is the first attempt at developing a method for the design of QSP-manufacturable composite structures, several simplifications are made to restrict the process versatility to a manageable level of complexity.

1. It is assumed that all patches are made from the same base ply material in order to reduce the design space.

2. Minimizing the amount of material waste during the cutting step is out of the scope of this work.

3. Devising the shape and position of the patches in the patch assembly is a complex task that is not fully completed in the present work. The proposed method returns an optimized partition of the structure into a reduced number of constant-stiffness areas for which feasible laminates are retrieved. The work focuses on the search for efficient trade-offs between the complexity of the partition of the structure and the mechanical performances of the optimized part.

4. The influence of process specific features such as cuts and thickness transitions on the strength of the composite structures are not considered. Instead, the present work focuses on stiffness-related design criteria.

5. Patch sliding occurring due to the flow of the matrix during thermoforming is neglected. Nonetheless, material orientation variations induced by the forming have to be taken into account in the design process for complex part shapes.

\section{The Quilted Stratum Design (QSD) method}

The design method is based on a three-step decomposition of the problem. The first step provides an idealized target design, relying on a gradient-based structural optimization and a homogenized description 
of the laminates. In the second step, the idealized continuous design obtained after the first step is simplified using a clustering algorithm to subdivide the structure into a small number of constant stiffness zones. Finally, the last step aims at retrieving the fiber orientations per layer and per zone.

During the first and second optimization steps, structural analyses are performed on the final thermoformed shape of the structure. By contrast, the third step is based on the flat perform. The forming of a $0^{\circ}$-ply is simulated using Altair Drape Estimator to estimate both the flat shape of the patch assembly and the spatial evolution of the material longitudinal direction on the final thermoformed shape. Structural optimizations are performed using Altair OptiStruct whilst the overall design approach is implemented in MATLAB.

\subsection{Step 1: structural optimization using lamination parameters}

In the first optimization step, thickness and stiffness distributions are used as design variables. Any mechanical response accessible through linear elastic FE analysis can be used either as a design objective or a constraint. The stiffness distribution is parameterized using lamination parameters [35]. Lamination parameters provide a compact and continuous parameterization of the stiffness behavior of composite laminates. The number of lamination parameters, twelve for the general case, is independent of the number of plies. The number of lamination parameters falls to eight in the case of uncoupled laminates (with the coupled stiffness matrix $\mathbf{B}=\mathbf{0}$ ) and to four in the case of uncoupled laminates with orthotropic membrane $\mathbf{A}$ and bending $\mathbf{D}$ properties. Furthermore, the design domain for lamination parameters is continuous and convex, making it suitable for gradient-based optimization. Individually, lamination parameters can take any value in the interval $[-1,1]$. They are, however, not independent since they are computed from a given stacking sequence. Deriving compact sets of feasibility constraints for the lamination parameters space has attracted the attention of many researchers since the first studies using lamination parameters as design variables for laminate optimization and different sets of equations are available depending on the number of non-zero parameters [21, 22, 25, 36-38].

The membrane stiffness matrix $\mathbf{A}$ and the bending stiffness matrix $\mathbf{D}$ are defined as linear functions of the lamination parameters:

$$
\begin{aligned}
& \mathbf{A}=h\left(\Gamma_{0}+\Gamma_{1} V_{1}^{A}+\Gamma_{2} V_{2}^{A}+\Gamma_{3} V_{3}^{A}+\Gamma_{4} V_{4}^{A}\right), \\
& \mathbf{D}=\frac{h^{3}}{12}\left(\Gamma_{0}+\Gamma_{1} V_{1}^{D}+\Gamma_{2} V_{2}^{D}+\Gamma_{3} V_{3}^{D}+\Gamma_{4} V_{4}^{D}\right) .
\end{aligned}
$$

where $h$ is the thickness of the laminate and $\Gamma_{i=1, \ldots, 4}$ are matrices dependent on the base ply in-plane stiffness properties. The membrane lamination parameters $V_{i=1, \ldots, 4}^{A}$ and bending lamination parameters $V_{i=1, \ldots, 4}^{D}$ are defined as follows:

$$
\begin{aligned}
& \left(V_{1}^{A}, V_{2}^{A}, V_{3}^{A}, V_{4}^{A}\right)=\int_{-1 / 2}^{1 / 2}(\cos 2 \theta(\bar{z}), \sin 2 \theta(\bar{z}), \cos 4 \theta(\bar{z}), \sin 4 \theta(\bar{z})) \mathrm{d} \bar{z}, \\
& \left(V_{1}^{D}, V_{2}^{D}, V_{3}^{D}, V_{4}^{D}\right)=\int_{-1 / 2}^{1 / 2} \bar{z}^{2}(\cos 2 \theta(\bar{z}), \sin 2 \theta(\bar{z}), \cos 4 \theta(\bar{z}), \sin 4 \theta(\bar{z})) \mathrm{d} \bar{z},
\end{aligned}
$$

where $\bar{z}$ is the normalized through the thickness dimension and $\theta(\bar{z})$ is the laminate stacking sequence. 


\subsection{Step 2: zone clustering}

The total number of design variables at step 1 depends on the assumptions made on the stiffness behavior of the laminate (which are closely related to laminate design guidelines such as symmetry and balance), the use of constant-thickness or variable-thickness laminates, and the desired type of thickness variations, which is closely related to the chosen manufacturing process. In the case of steered laminates, continuous stiffness variations over the structure are possible which can be described using a set of design variables per element of the model. In the case of more conventional variable-thickness straight-fiber laminates, the ply-drop locations are usually predefined and design variables are assigned per constant-stiffness areas. The design of QSP laminates constitutes an intermediate class of problems. The process does not enable continuous variations of the laminate stiffness and the subdivision of the structure into constant stiffness areas is not known a priori.

From a manufacturing viewpoint, transitions between constant stiffness areas are obtained by terminating patches or joining patches with different orientations. The smoother the stiffness variations over the structure, the smaller and more numerous are the patches and the more complicated is the patch assembly. In Step 1, a first structural optimization is performed using a fine initial subdivision of the structure into constant-stiffness zones. For small FE models, finite elements can directly be used as zones. For larger models, however, elements are grouped into clusters to limit the number of design variables and feasibility constraints. The optimization result can be interpreted as the best achievable solution using the smallest possible patch size. The zoning of the structure is simplified in Step 2 to search for an efficient trade-off between the simplicity of the patch assembly and the performance of the structure. Clusters of zones are formed based on the structural similarities between each zone employing a $k$-means clustering method [39]. Each zone is represented as a vector containing the optimal values of the corresponding design variables (e.g. thickness and lamination parameters) and the coordinates of the center of the zone in order to promote the formation of clusters of adjacent zones.

The $k$-means clustering finds a partition in which elements within a cluster are closer to the cluster centroid than to any other cluster centroid. A heuristic is used to find the centroids that maximize the separation between the clusters. The optimal number of clusters $k$ can be determined by experimenting with a limited range of values for $k$. In this work, the average silhouette value is used as a quantitative indicator of how well-separated the clusters are [40]. The best values of $k$ tend to maximize the average silhouette value. The corresponding clusters form a meaningful partition of the structure with respect to the optimal stiffness distribution. However, in most practical cases, perfect separation between neighboring clusters cannot be achieved. User intervention is often required to check and eventually adjust the clusters. Once the number of clusters chosen and the clusters validated, a simplified zoning of the structure is defined. Each cluster is attributed a single set of design variables and a second structural optimization is run to determine the optimal thickness and lamination parameters distributions and ensure that the performance loss with respect to the first optimal design is acceptable. The feasible lamination parameters space being convex, the average values of the first optimization design variables over each cluster can be used as a starting point for the 
second optimization run.

\subsection{Step 3: patch assembly retrieval}

Step 2 results in a simplified zoning of the part, with optimal membrane and bending stiffness matrices per zone. In Step 3 the laminates that best matches the target stiffness distribution are retrieved. The QSP allows to join patches of different material orientation end to end. Thus, within a single layer, no fiber continuity constraint applies with the QSP. Therefore, a first solution can be obtained by searching for the best laminate in each zone considered separately. In the present work, independent stiffness matching optimizations are performed for each zone. Proximity between a laminate, with membrane stiffness matrix $\mathbf{A}$ and bending stiffness matrix $\mathbf{D}$, and the target stiffness matrices $\mathbf{A}^{*}$ and $\mathbf{D}^{*}$ is assessed using the A-distance $d_{A}$ and D-distance $d_{D}$ values [41]:

$$
\begin{aligned}
& d_{A}\left(\mathbf{A}, \mathbf{A}^{*}\right)=\operatorname{Tr}\left(\mathbf{A}^{-1} \mathbf{A}^{*}\right)+\operatorname{Tr}\left(\mathbf{A} \mathbf{A}^{*-1}\right)-6, \\
& d_{D}\left(\mathbf{D}_{1}, \mathbf{D}^{*}\right)=\operatorname{Tr}\left(\mathbf{D}^{-1} \mathbf{D}^{*}\right)+\operatorname{Tr}\left(\mathbf{D D}^{*-1}\right)-6 .
\end{aligned}
$$

The weighting between $d_{A}$ and $d_{D}$ depends on the application, nevertheless giving the same weight to both criteria is usually good practice.

Laminate stiffness matching optimization can be performed using specialized algorithms. However, with the QSP, the number of layers in the patch assembly is limited. On the one hand, the laminate total thickness is limited due to process-related constraints for the final thermoforming phase. Laminates more than $10 \mathrm{~mm}$ thick would be very difficult to heat correctly in a limited time. On the second hand, each layer is laid by a dedicated assembly station. Thus the number of layers of the patch assembly drives the design and cost of the scalable assembly system. Last but not least, thick plies are generally less expensive than thin plies and will be preferred to minimize the cost of the parts. Material cost is indeed one of the main cost driver for mass production. All the above considerations tend to reduce the number of layers, usually bellow 30 layers. With limited sets of allowed ply orientations, such as $0^{\circ}, \pm 45^{\circ}, 90^{\circ}$, the problem becomes tractable for database enumeration. Using a database of laminates also simplifies the enforcement of laminate design guidelines such as symmetry, balance, continuity of the surface plies or $10 \%$ design guideline.

\section{Applications}

Two applications of the QSD method are presented and discussed in this Section. The first application serves as a demonstrative academic benchmark case study for the proposed design method, showcasing its potential and efficiency. The second application, industrially-focused, illustrates the optimization of a more complex automotive part, targeting a reduction in mass.

\subsection{Buckling optimization of a square plate}

The first application deals with the buckling optimization of a square plate under uniaxial compression, a problem previously studied by IJsselmuiden et al. [23]. All four edges are simply supported and remain 
straight. Furthermore, the orthotropic material properties are $E_{11}=181 \mathrm{GPa}, E_{22}=10.3 \mathrm{GPa}, G_{12}=7.17$ GPa and $v_{12}=0.28$. The laminate is assumed to be symmetric and balanced and the total number of plies is set to 16 with a total thickness of $1.524 \mathrm{~mm}$. The plate is modeled in OptiStruct, using a square mesh of $20 \times 20$ quadrangular shell elements.

Step 1 of the optimization is performed using an independent set of design variables per element: the lamination parameters $V_{1}^{A}$ and $V_{3}^{A}$ for the membrane behavior and $V_{1}^{D}$ and $V_{3}^{D}$ for bending. The optimized lamination parameters distributions are presented in Figure 2, which shows obvious similarities with the results previously reported in the literature [23]. Despite some differences, especially in $V_{3}^{A}$ and $V_{3}^{D}$ distributions, the optimized buckling load, normalized with respect to the "black aluminum" solution (i.e. all lamination parameters equal to zero), matches the one reported in [23]. That is, the buckling load of the variable stiffness (VS) solution is about 2.53 times higher than the quasi-isotropic solution.

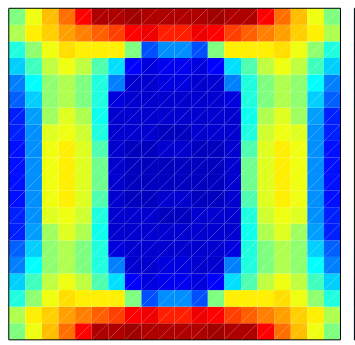

$V_{1}^{A}$

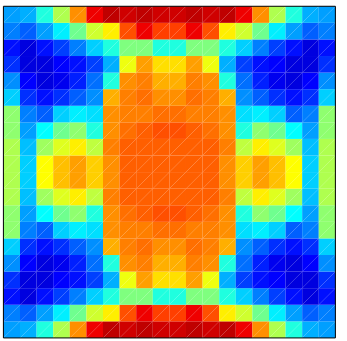

$V_{3}^{A}$

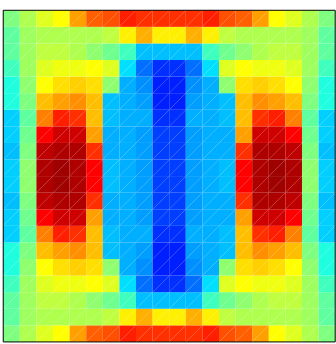

$V_{1}^{D}$

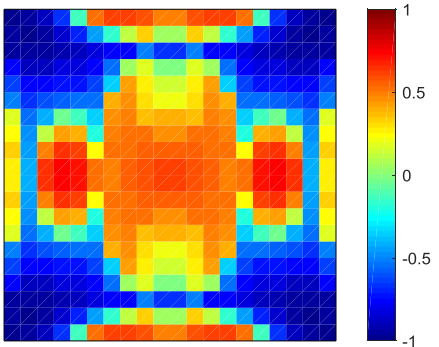

$V_{3}^{D}$

Figure 2: Step1. Optimal lamination parameters distributions.

In Step 2, the plate is partitioned into constant stiffness (CS) areas, base on the results of Step 1. Several clustering attempts are performed using the $k$-means algorithm for different numbers of clusters. In the VS plate the buckling improvement with respect to CS design is related to load redistribution induced by the spatial variation of the membrane stiffness properties. Thus, in the present case, the clustering is performed based on the position of the center of the elements and on the optimal values of the membrane lamination parameters $V_{1}^{A}$ and $V_{3}^{A}$ obtained from Step 1. The evolution of the mean silhouette value (MSV) as a function of the number of clusters is plotted in Figure 3 . The maximal MSV value is reached for 7 clusters, but the curve shows that partition of similar quality can be obtained for 3 or 5 clusters. The corresponding partitions of the plate are shown in Figure 4. The 3-cluster solution gives a simple and seemingly relevant partitioning of the structure. It is interesting to note that clusters can cover disconnected regions of the part. Hence, from a process-related point of view, a cluster can correspond to several patches. The same comment applies to the 5-cluster solution. However, this solution is much less relevant since cluster 1 is far too dispersed. Such a solution would require a manual correction. The solution with 7 clusters presents transition zones between a few main clusters that are very similar to the ones of the 3-cluster solution.

In order to assess the influence of clustering on the mechanical behavior, a lamination parameter based 


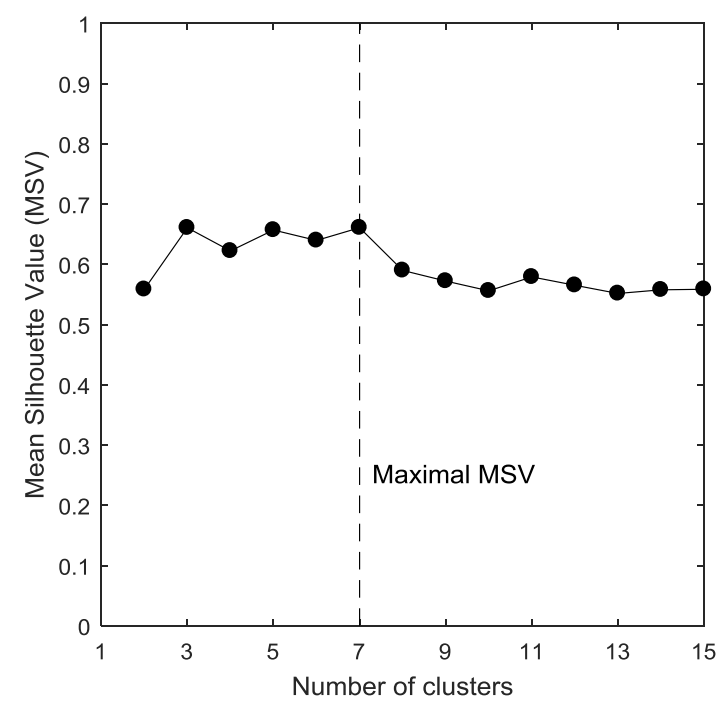

Figure 3: Step2. Evolution of the Mean Silhouette Value as a function of the number of clusters.

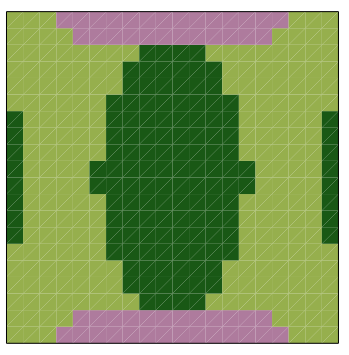

3 clusters
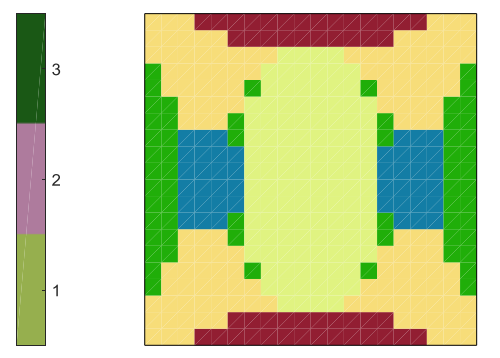

5 clusters

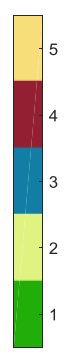

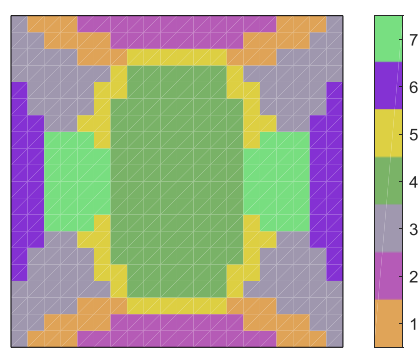

7 clusters

Figure 4: Step2. Clusterization examples. The clusters are identified by the colorbars.

optimization is performed for each number of clusters $k$. The optimal buckling load is plotted as a function of $k$ in Figure 5 For $k=1$, the solution corresponds to the best CS laminate, i.e. the $\pm 45^{\circ}$ laminate. The curve quickly converges towards the VS solution. For $k=3$, the solution is only $10 \%$ below the VS solution.

Independent laminate retrieval per cluster is performed at Step 3. Here, the distance to the stiffness target is evaluated for all 81 laminates $\left[ \pm \theta_{1} / \pm \theta_{2} / \pm \theta_{3} / \pm \theta_{4}\right]_{s}$, with $\theta_{i=1, \ldots 4}$ taking value in the set $\left\{0^{\circ}, 45^{\circ}, 90^{\circ}\right\}$. For each cluster, the laminate minimizing the distance to the stiffness target is chosen. The buckling load difference between the simplified continuous solution from Step 2 and the layered solution from Step 3 is shown in Figure 5 for $k$ ranging from 1 to 15 . The buckling load of the layered solutions reaches a plateau at $k=3$. More detailed partitioning of the plate with larger numbers of clusters $k$ brings no improvement in the buckling load. Direct application of Step 3 on the VS solution form Step 1 returns a solution $(k=400)$ whose buckling load $\lambda / \lambda_{0}=1.69$ is consistent with the plateau observed in Figure 5. This plateau is 


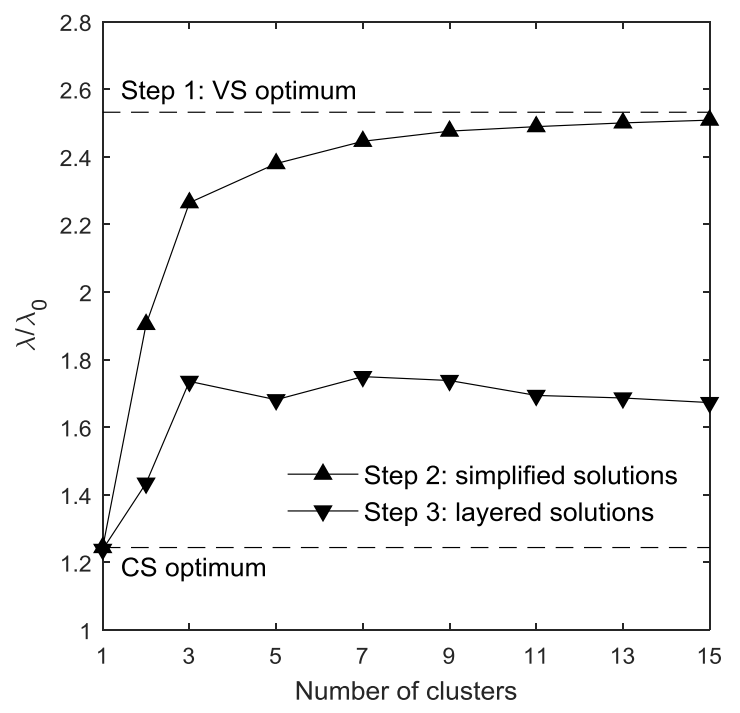

Figure 5: Evolution of the critical buckling factor as a function of the number of clusters. Results are presented for each step of the proposed approach. All buckling factors are normalized with respect to $\lambda_{0}$ the buckling load the "black aluminum" solution. Step 1 and Step 2 solutions correspond to stiffness distributions. Laminates are known for Step 3 solutions.

due to the low number of possible laminates evaluated at step 3. Both the angular discretization and the thickness discretization due to the use of a single base ply prevent accurate matching of the target stiffness distributions. These results show that a simple partitioning of the structure is enough to obtain a significant improvement over the CS designs (about $73 \%$ improvement with respect to the "black aluminum solution" and $10 \%$ with respect to the CS optimum.

The four design layers of the 3-cluster solution are detailed in Figure 6, For each layer, the elements are grouped per material orientation. The groups of elements sketch the shape of the patches. For example, layer 1 can be covered using a single patch with material orientation $45^{\circ}$. Layers 3 and 5 could be covered using 2 patches at $0^{\circ}, 2$ patches at $45^{\circ}$ and 3 patches at $90^{\circ}$. At this stage of the design process, however, further work is required to obtain a complete definition of the patches. The patch edges at Step 3 are defined by the exterior edges of the element sets and should be simplified without being constrained by the FE mesh. Additionally, in the proposed design method, patch edges correspond to the boundaries of the constant stiffness areas obtained after simplification (i.e. Step 2). Therefore the final design will display clear thickness discontinuities at the interface between patches, resulting in weak areas subject to failure. However, as previously mentioned in Section 2, the present work focuses on stiffness-based criteria and the development of a post-processing algorithm for the design of smooth transition between patches is beyond the scope of this work.

Note that the calculation time at Step 1 is about 3 minutes on a regular laptop (4 Intel Core i7-37440QM CPUs and 8 GB of RAM). The overall calculation time for Step 2 and 3, including application of the clustering algorithm for $k$ ranging from 1 to 15 , continuous optimization of a simplified solution for a 
chosen number of clusters $k$ and finally laminate retrieval for this design is less than 5 minutes.

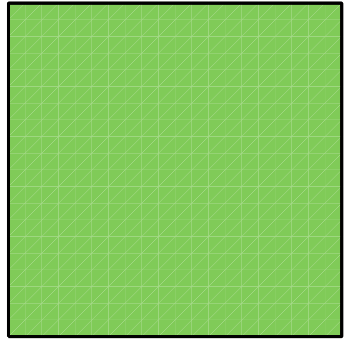

Layer $1\left(\theta_{1}\right)$

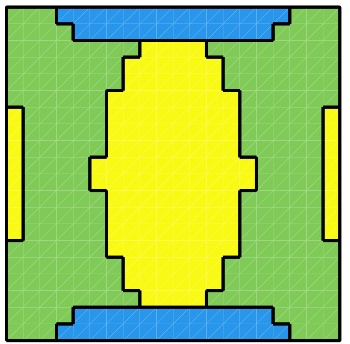

Layer $3\left(\theta_{2}\right)$

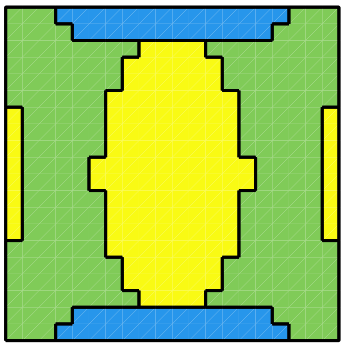

Layer $5\left(\theta_{3}\right)$

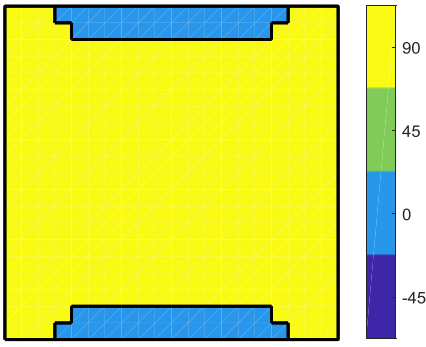

Layer $7\left(\theta_{4}\right)$

Figure 6: Step3. patch assembly for the solution with 3 clusters. Layers 1 is on the surface of the laminate. Layer 7 is close to the midplane.

\subsection{Mass minimization of an automotive composite suspension arm}

The second application deals with the mass minimization of a car suspension arm under nodal displacements constraints. The FE model of the suspension arm is shown in Figure 7. The wishbone is made of two symmetrical shells welded together along the plane $(\mathrm{O}, \mathrm{x}, \mathrm{y})$. Points $\mathrm{O}$ and $\mathrm{A}$ correspond to the junction of the part with the car chassis. Point B corresponds to the junction with the wheel. Ball joints are used at all three points, thus no bending moments are transferred to the part. The double composite shell geometry provides very high bending stiffness to the part so that global buckling is not a concern. Thus only half of the part is modeled and a symmetry condition is enforced on the $(\mathrm{O}, \mathrm{x}, \mathrm{y})$ plane. The load F corresponds to a critical design case with respect to the wheel displacement. At point $\mathrm{B}$, the translation along the $\mathrm{x}$-direction is constrained by an upper bound $U_{x}(B)^{U B}$ and the translation along the y-direction is constrained by a lower bound $U_{y}(B)^{L B}$. The part is made from a T700S/PA66 thermoplastic material with elastic properties $E_{11}=$ 104.2 GPa, $E_{22}=5450 \mathrm{MPa}, G_{12}=2022 \mathrm{GPa}$ and $v_{12}=0.34$ (see [42]). The tape thickness is $0.316 \mathrm{~mm}$.

The reference solution is a 12-ply constant stiffness laminate with quasi-isotropic membrane properties. The mass of the part is about $m_{0}=500 \mathrm{~g}$. The aim of the optimization is to design a feasible solution with a mass reduced by $20 \%$. The first step optimization problem is formulated as follows. The original mass minimization problem with constrained displacements at point B is converted into the minimization of an elliptic function of the displacements at point B under constrained mass, better suited to a stiffness-matching approach.

$$
\begin{aligned}
& \text { minimize }\left(\frac{U_{x}(B)}{U_{x}^{U} B(B)}\right)^{2}+\left(\frac{U_{y}(B)}{U_{y}^{L} B(B)}\right)^{2}, \\
& \text { submitted to } m \leq 0.8 \times m_{0} .
\end{aligned}
$$

The design variables are the laminate thickness $T$ and the lamination parameters $V_{1}^{A}$ and $V_{3}^{A}$ for the membrane behavior and $V_{1}^{D}$ and $V_{3}^{D}$ for the bending behavior. The laminate thickness ranges from $2.528 \mathrm{~mm}$ (8 plies) to $5.056 \mathrm{~mm}$ (16 plies). The FE model of the part has about 10,000 shell elements. Using a set of 


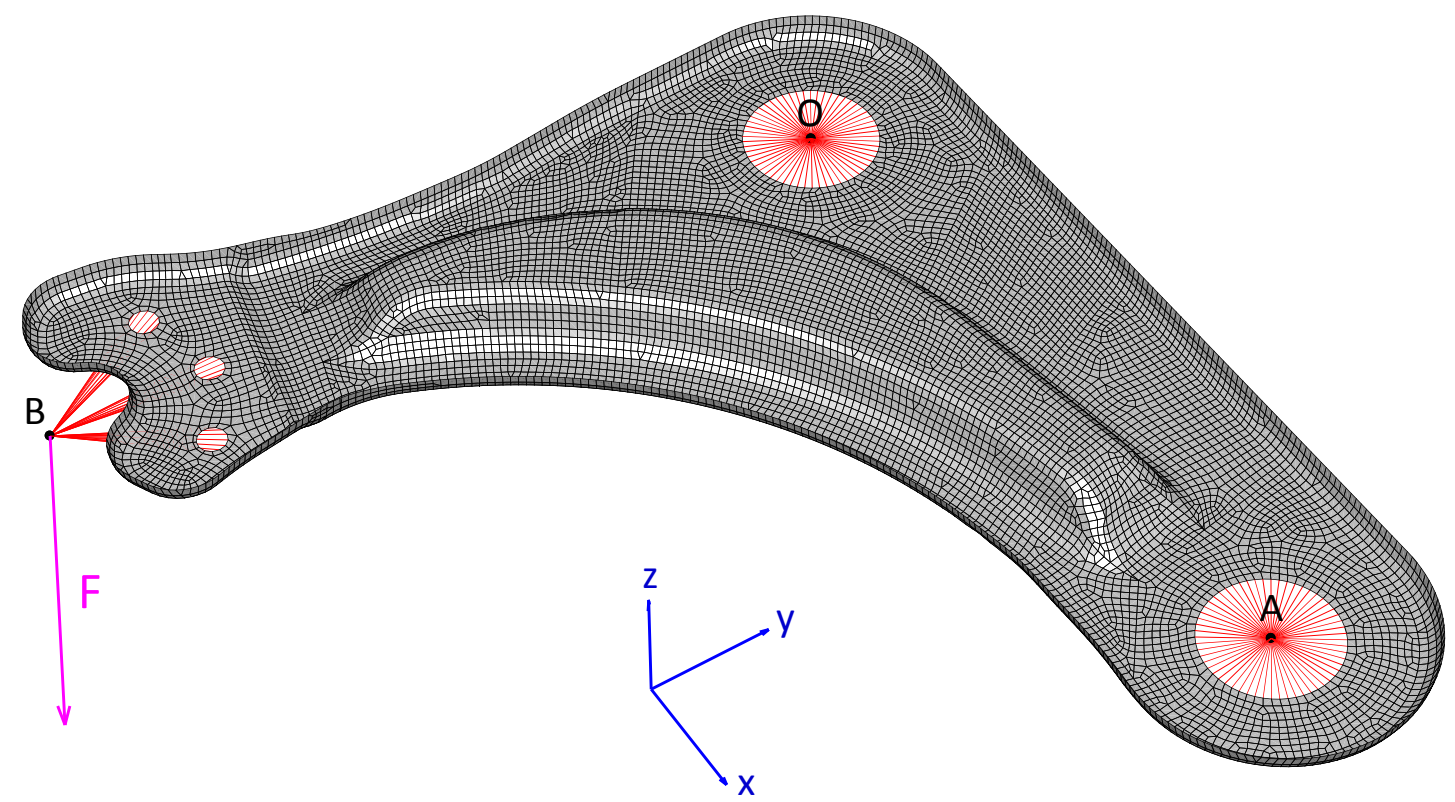

Figure 7: Mesh and loading conditions for the FE model of the upper half of the automotive wishbone. The plane $(\mathrm{O}, \mathrm{x}, \mathrm{y})$ is a plane of symmetry. Points A and B belong to that plane as well as the load vector F. All translations are blocked at point O. Translations along the $\mathrm{y}$-direction and z-direction are blocked at point A. Translation along the z-direction is blocked at point B.

design variables per element at Step 1 would result in a very large number of design variables and feasibility constraints. This seems quite irrelevant with respect to Step 2 which aims at reducing as much as possible the complexity of the optimal thickness and stiffness distributions from Step 1. In order to reduce the number of design variables and the calculation cost, elements are grouped into zones of uniform stiffness properties using the $k$-means algorithm applied to the 3D coordinates of the centroid of the elements. Here $k$ is determined by specifying a characteristic diameter for the zones, supposing they are circular. In the present case, a $20 \mathrm{~mm}$ diameter results in 292 zones. The resulting zones are somewhat similar to Voronoï cells. The optimal thickness and lamination parameters distributions after Step 1 are shown in Figure 8
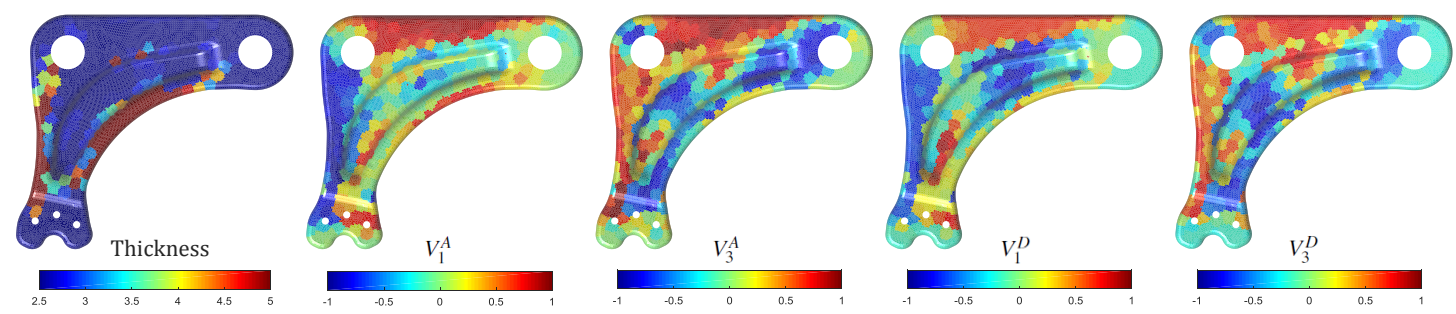

Figure 8: Step1. Optimal design variables distributions.

A simplified partition of the part is built at Step 2, based on the optimum solution achieved at Step 1. The maximal average silhouette value is obtained using 6 clusters. The partition is manually corrected to form compact clusters of zones. A seventh cluster is added in order to enforce constant stiffness around the hole 
centered on point $\mathrm{O}$. The simplified partition is shown in Figure 9 The figure also presents the optimal design variables distributions after continuous optimization on the simplified partition of the part. Using the simplified partition enables to filter the irregularities observed on the thickness distribution in Figure 8 Zones 3 and 4 reproduce features that are clearly observable in the distributions of the lamination parameters values in Figure 8 . Zone 3 corresponds to a laminate mostly oriented in the $90^{\circ}$ direction $\left(V_{1}^{A}<-0.8\right.$ and $\left.V_{3}^{A}>0.6\right)$ while Zone 4 is mostly oriented in the $0^{\circ}$ direction $\left(V_{1}^{A}>0.9\right.$ and $\left.V_{3}^{A}>0.9\right)$. Zone 6 is directly inherited from the thickness distribution shown in Figure 8 . The remaining zones tend to average the variations observed in the distributions of the lamination parameters values after Step 1. Note that Step 2 optimization has been restarted after convergence to enforce discrete thickness values corresponding to integer number of plies.
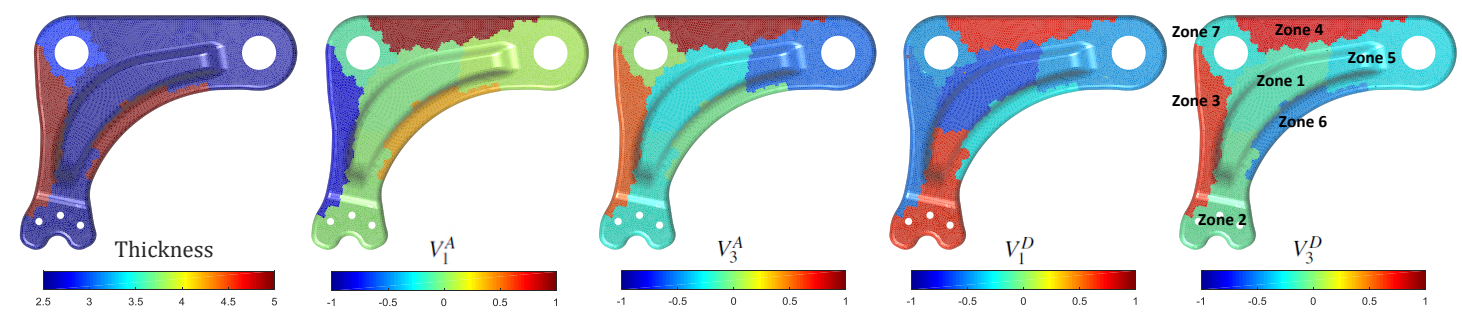

Figure 9: Step2. Optimal design variables distributions.

Balanced laminates are retrieved in Step 3. Note that the overall symmetry of the double-shell part allows to use non-symmetrical laminates A first layered design (solution 1) is retrieved with no additional design guideline enforced. A second solution (solution 2) is retrieved with a $\left(0^{\circ} / 90^{\circ}\right)$ pair of plies on the bottom surface of the part and a $\left( \pm 45^{\circ}\right)$ pair of plies on the top surface. Solutions 1 and 2 are compared with Step 2 solution in Figures 10 and 11 respectively. The figures show the polar evolution of the apparent membrane stiffness $A_{x x}$ and bending stiffness $D_{x x}$ per zone. Table 1 summarizes the results for all three optimization steps. Figure 10 shows that solution 1 is a close match of Step 2 continuous optimum. Due to the prescribed ply orientations on the surfaces of the part, solution 2 is farther from the target, especially in terms of bending stiffness. Table 1 shows that the displacements at point $\mathrm{B}$ are very sensitive to small stiffness variations. However, both layered solutions obtained at Step 3 significantly reduce the magnitude of the displacement at point B with a $20 \%$ mass saving. Step 3 solutions are detailed in Table 2 


\begin{tabular}{lccc}
\hline Solution & $\frac{U_{x}(B)}{U_{x}^{U B}(B)}$ & $\frac{U_{y}(B)}{U_{y}^{L I B}(B)}$ & Mass [g] \\
\hline Reference CS design & 0.87 & 0.88 & 500 \\
Step 1 & 0.67 & 0.15 & 392 \\
Step 2 & 0.76 & 0.26 & 400 \\
Step 3 (solution 1) & 0.83 & 0.34 & 400 \\
Step 3 (solution 2) & 0.89 & 0.53 & 400 \\
\hline
\end{tabular}

Table 1: Overview of the designs obtained after each optimization step, compared with the reference CS design $\left[(90 / 45 / 0 /-45)_{3}\right]_{s}$.

\begin{tabular}{|c|c|}
\hline Zone & Solution 1 \\
\hline 1 & 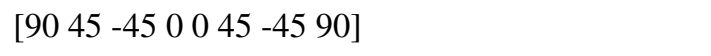 \\
\hline 2 & 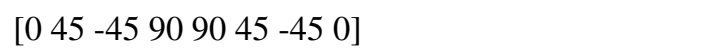 \\
\hline 3 & [90 $9045909090909090909090-459090$ 0] \\
\hline 4 & {$\left[\begin{array}{llllllll}0 & 45 & 0 & 0 & 0 & 0 & -45 & 0\end{array}\right]$} \\
\hline 5 & {$\left[\begin{array}{llllllllllllllll}-45 & 45 & 45 & 0 & -45 & -45 & 45 & 90\end{array}\right]$} \\
\hline 6 & 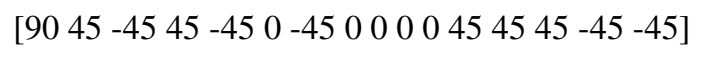 \\
\hline 7 & [-45 $4590-45000045$ 90] \\
\hline Zone & Solution 2 \\
\hline 1 & 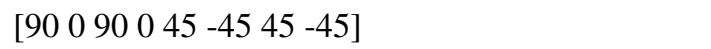 \\
\hline 2 & 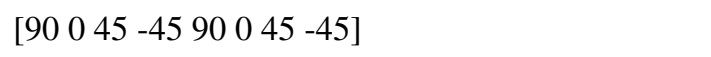 \\
\hline 3 & [90 $090090090045-4590090045$-45] \\
\hline 4 & 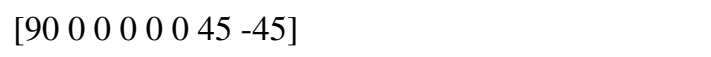 \\
\hline 5 & 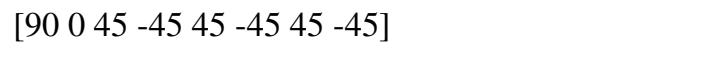 \\
\hline 6 & 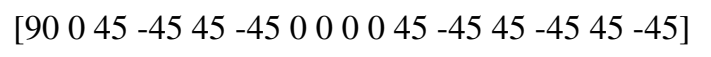 \\
\hline 7 & [90 $09090045-4545$-45] \\
\hline
\end{tabular}

Table 2: Step 3. Laminate per zone for solution 1 and solution 2. 


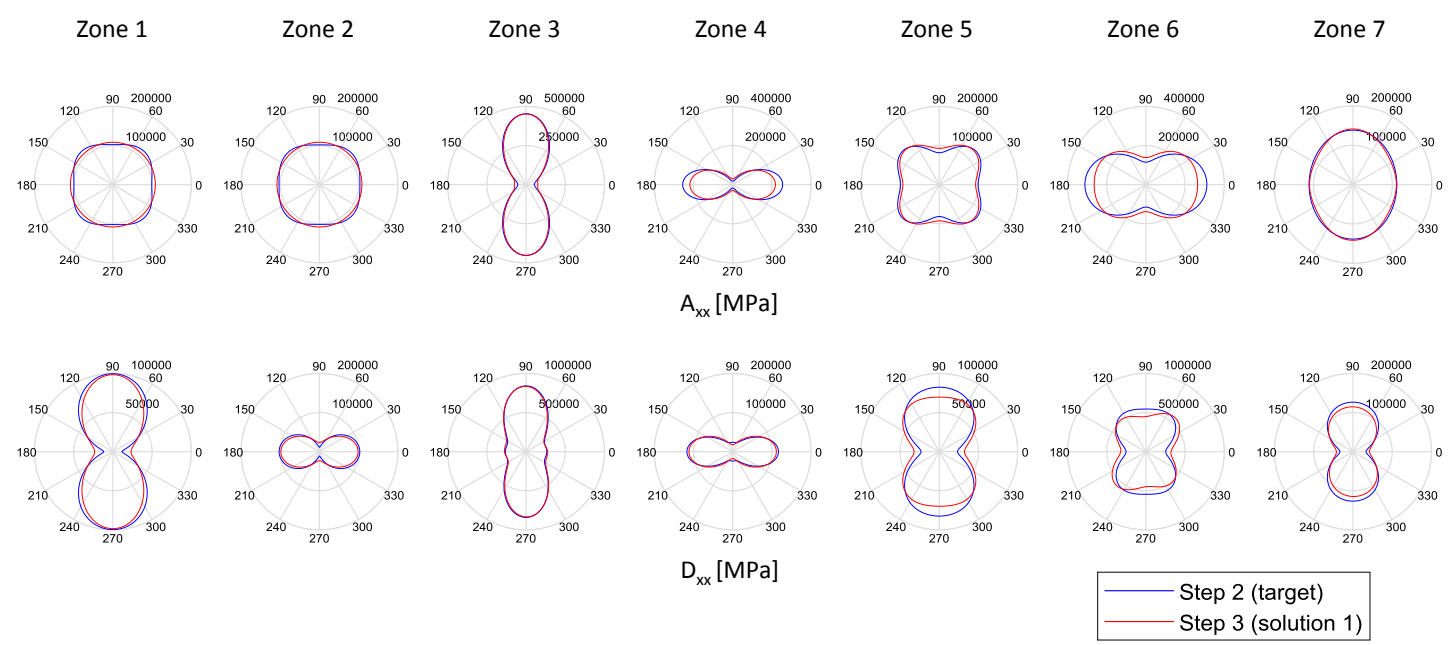

Figure 10: Step3. Solution 1. Stiffness matching with balanced laminates. Allowed ply orientations are $0^{\circ}, \pm 45^{\circ}, 90^{\circ}$.

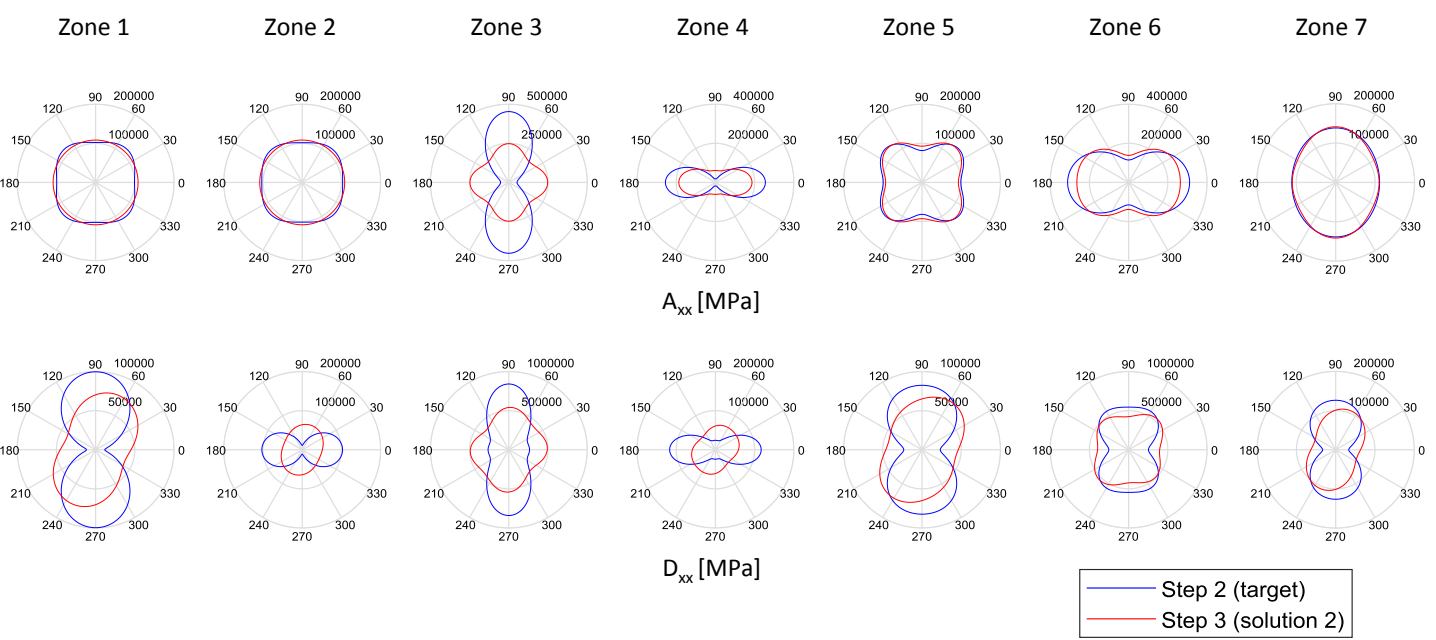

Figure 11: Step3. Solution 2. Stiffness matching with balanced laminates. Prescribed $\left(0^{\circ} / 90^{\circ}\right)$ pair of plies on the bottom surface of the part and a $\left( \pm 45^{\circ}\right)$ pair of plies on the top surface. Allowed ply orientations are $0^{\circ}, \pm 45^{\circ}, 90^{\circ}$.

\section{Concluding Remarks}

The work presented in this paper is a first step towards a composite structure design method specialized for the Quilted Stratum Process. The main challenge was to bring together the great versatility of composite designs offered by the QSP under a single coherent framework, automating and simplifying the design of QSP-manufactured structures for future applications. To this end, the Quilted Stratum Design (QSD) method was proposed. The QSD is a three-step optimization procedure during which the structure is first ideally optimized, then simplified and finally converted into a layered design that can be interpreted for QSP-manufacturing.

The proposed QSD method was successfully applied to both an academic and an industrial application case 
study. The first level optimization provides ideally optimized variable-thickness and variable-stiffness structural designs used both as starting points and references for the subsequent optimization steps. Considering the QSP manufacturing constraints and the inherent manufacturing complexity of continuously varying thickness and stiffness distributions, partitioning the structure into constant stiffness zones was found to provide good trade-off designs, significantly reducing the structure complexity at the cost of a small reduction in performance. In both our applications, a small number of zones was found to be sufficient to significantly over-perform conventional constant-stiffness designs and well-compatible with the QSP.

With the current state of the method, the solution is described by independent laminates for each constant stiffness areas. Future work should investigate how to retrieve a detailed description of the patch assembly at Step 3. Two main points have to be taken into account. First, the shape of the patches have to be constrained in order to minimize material wastes during the cutting phase, since for high volume production material waste drives the overall cost of the composite part. Second, discontinuities at the interfaces between patches create weak areas subject to failure. Strength related design guidelines have to be introduced in Step 3 in order to design efficient patch overlapping schemes with respect to failure and avoid matrix-dominated failure modes driven by the coalescence of patch delamination and matrix cracks at the junction between patches.

\section{References}

[1] Hossein Ghiasi, Damiano Pasini, and Larry Lessard. Optimum stacking sequence design of composite materials Part I: Constant stiffness design. Composite Structures, 90(1):1-11, September 2009.

[2] Hossein Ghiasi, Kazem Fayazbakhsh, Damiano Pasini, and Larry Lessard. Optimum stacking sequence design of composite materials Part II: Variable stiffness design. Composite Structures, 93(1):113, December 2010.

[3] MIL-HDBK-17-1F: Composite Materials Handbook, Volume 1 - Polymer Matrix Composites Guidelines for Characterization of Structural Materials. U.S. Department of Defense, December 2002.

[4] J. A. Bailie, R. P. Ley, and A. Pasricha. A summary and review of composite laminate design guidelines. Technical report NASA NAS1-19347, 1997.

[5] Rodolphe Le Riche and Raphael T. Haftka. Optimization of laminate stacking sequence for buckling load maximization by genetic algorithm. AIAA Journal, 31(5):951-956, May 1993.

[6] S. Nagendra, Raphael T. Haftka, and Zafer Gürdal. Design of a blade stiffened composite panel by a genetic algorithm. In Proc. 34th. Structural Dynamics and Materials Conf., Lajolla, CA, pages 2418-2436, 1993. 
[7] Boyang Liu, Raphael T. Haftka, Mehmet A. Akgün, and Akira Todoroki. Permutation genetic algorithm for stacking sequence design of composite laminates. Computer Methods in Applied Mechanics and Engineering, 186(2-4):357-372, June 2000.

[8] Birna P. Kristinsdottir, Zelda B. Zabinsky, Mark E. Tuttle, and Sudipto Neogi. Optimal design of large composite panels with varying loads. Composite Structures, 51(1):93-102, 2001.

[9] Boyang Liu and Raphael Haftka. Composite wing structural design optimization with continuity constraints. American Institute of Aeronautics and Astronautics, June 2001.

[10] Grant Soremekun, Zafer Gürdal, Christos Kassapoglou, and Darryl Toni. Stacking sequence blending of multiple composite laminates using genetic algorithms. Composite Structures, 56(1):53-62, 2002.

[11] David B. Adams, Layne T. Watson, Zafer Gürdal, and Christine M. Anderson-Cook. Genetic algorithm optimization and blending of composite laminates by locally reducing laminate thickness. Advances in Engineering Software, 35(1):35-43, January 2004.

[12] F.-X. Irisarri, A. Lasseigne, F.-H. Leroy, and R. Le Riche. Optimal design of laminated composite structures with ply drops using stacking sequence tables. Composite Structures, 107:559-569, January 2014.

[13] S. Zein, P. Basso, and S. Grihon. A constraint satisfaction programming approach for computing manufacturable stacking sequences. Computers \& Structures, 136:56-63, May 2014.

[14] M. W. Hyer and R. F. Charette. Use of curvilinear fiber format in composite structure design. AIAA Journal, 29(6):1011-1015, June 1991.

[15] Zafer Gürdal, Brian Tatting, and K. Wu. Tow-Placement Technology and Fabrication Issues for Laminated Composite Structures. American Institute of Aeronautics and Astronautics, April 2005.

[16] Z. Gürdal, B.F. Tatting, and C.K. Wu. Variable stiffness composite panels: Effects of stiffness variation on the in-plane and buckling response. Composites Part A: Applied Science and Manufacturing, 39(5):911-922, May 2008.

[17] C.S. Lopes, Z. Gürdal, and P.P. Camanho. Tailoring for strength of composite steered-fibre panels with cutouts. Composites Part A: Applied Science and Manufacturing, 41(12):1760-1767, December 2010.

[18] Zhangming Wu, Paul M. Weaver, Gangadharan Raju, and Byung Chul Kim. Buckling analysis and optimisation of variable angle tow composite plates. Thin-Walled Structures, 60:163-172, November 2012.

[19] Byung Chul Kim, Kevin Potter, and Paul M. Weaver. Continuous tow shearing for manufacturing variable angle tow composites. Composites Part A: Applied Science and Manufacturing, 43(8):13471356, August 2012. 
[20] Marco Montemurro and Anita Catapano. On the effective integration of manufacturability constraints within the multi-scale methodology for designing variable angle-tow laminates. Composite Structures, 161:145-159, February 2017.

[21] Mitsunori Miki and Yoshihiko Sugiyamat. Optimum Design of Laminated Composite Plates Using Lamination Parameters. AIAA Journal, 31(5):921-922, May 1993.

[22] J.L. Grenestedt and P. Gudmundson. Layup Optimization of Composite Material Structures. In Optimal Design with Advanced Materials, pages 311-336. Elsevier, 1993.

[23] S. T. IJsselmuiden, Mostafa M. Abdalla, and Zafer Gürdal. Optimization of Variable-Stiffness Panels for Maximum Buckling Load Using Lamination Parameters. AIAA Journal, 48(1):134-143, January 2010 .

[24] Dianzi Liu and Vassili V. Toropov. A lamination parameter-based strategy for solving an integercontinuous problem arising in composite optimization. Computers \& Structures, 128:170-174, November 2013.

[25] Zhangming Wu, Gangadharan Raju, and Paul M. Weaver. Framework for the Buckling Optimization of Variable-Angle Tow Composite Plates. AIAA Journal, 53(12):3788-3804, December 2015.

[26] P. Vannucci and G. Verchery. Stiffness design of laminates using the polar method. International Journal of Solids and Structures, 38(50-51):9281-9294, December 2001.

[27] A. Jibawy, C. Julien, B. Desmorat, A. Vincenti, and F. Léné. Hierarchical structural optimization of laminated plates using polar representation. International Journal of Solids and Structures, 48(18):2576-2584, September 2011.

[28] Anita Catapano, Boris Desmorat, and Paolo Vannucci. Stiffness and Strength Optimization of the Anisotropy Distribution for Laminated Structures. Journal of Optimization Theory and Applications, December 2014.

[29] Franois-Xavier Irisarri, Daniël M.J. Peeters, and Mostafa M. Abdalla. Optimisation of ply drop order in variable stiffness laminates. Composite Structures, 152:791-799, September 2016.

[30] Adriana W. Blom, Mostafa M. Abdalla, and Zafer Gürdal. Optimization of course locations in fiberplaced panels for general fiber angle distributions. Composites Science and Technology, 70(4):564570, April 2010.

[31] Daniël M. J. Peeters, Simon Hesse, and Mostafa M. Abdalla. Stacking sequence optimisation of variable stiffness laminates with manufacturing constraints. Composite Structures, 125(0):596 - 604, 2015. 
[32] Lennart Wedhorn and Robert Ebeling. Quilted Stratum Process for High-performance CFRP Production. Lightweight Design worldwide, 10(1):50-55, 2017.

[33] N. Zehnder and P. Ermanni. A methodology for the global optimization of laminated composite structures. Composite Structures, 72(3):311-320, March 2006.

[34] M. Giger, D. Keller, and P. Ermanni. A graph-based parameterization concept for global laminate optimization. Structural and Multidisciplinary Optimization, 36(3):289-305, September 2008.

[35] Zafer Gurdal, Raphael T. Haftka, and Prabhat Hajela. Design and optimization of laminated composite materials. A Wiley-Interscience Publication. Wiley, New York, NY, 1999.

[36] Cezar Gabriel Diaconu, Masaki Sato, and Hideki Sekine. Feasible Region in General Design Space of Lamination Parameters for Laminated Composites. AIAA Journal, 40(3):559-565, March 2002.

[37] M. W. Bloomfield, C. G. Diaconu, and P. M. Weaver. On feasible regions of lamination parameters for lay-up optimization of laminated composites. Proceedings of the Royal Society A: Mathematical, Physical and Engineering Sciences, 465(2104):1123-1143, April 2009.

[38] T. Macquart, V. Maes, Marco T. Bordogna, A. Pirrera, and P.M. Weaver. Optimisation of composite structures Enforcing the feasibility of lamination parameter constraints with computationally-efficient maps. Composite Structures, 192:605-615, May 2018.

[39] D. Arthur and S. Vassilvitskii. k-means++: the advantages of careful seeding. In SODA '07: Proceedings of the eighteenth annual ACM-SIAM symposium on Discrete algorithms, pages 1027-1035. Society for Industrial and Applied Mathematics, 2007.

[40] Leonard Kaufman and Peter J. Rousseeuw. Finding groups in data: an introduction to cluster analysis. Wiley series in probability and mathematical statistics. Wiley, New York, 1990.

[41] Francois-Xavier Irisarri, Mostafa M. Abdalla, and Zafer Gürdal. Improved Shepard's Method for the Optimization of Composite Structures. AIAA Journal, 49(12):2726-2736, December 2011.

[42] Y. Todeschini, C. Huchette, C. Julien, and D. Espinassou. Analysis of damage and failure mechanisms of Quilted Stratum Process composite parts. In ECCM17, MUNICH, Germany, June 2016. 